\title{
Article
}

\section{Integrating Serum Biomarkers into Prediction Models for Biochemical Recurrence Following Radical Prostatectomy}

\author{
Shirin Moghaddam ${ }^{1,2, *,+}+$, Amirhossein Jalali ${ }^{1,2, *,+}$, Amanda $\mathrm{O}^{\prime} \mathrm{Neill}^{2}$, Lisa Murphy ${ }^{2}$, Laura Gorman ${ }^{2}$, \\ Anne-Marie Reilly ${ }^{2}$, Áine Heffernan ${ }^{2}$, Thomas Lynch ${ }^{3}$, Richard Power ${ }^{4}$, Kieran J. O'Malley ${ }^{5}$, \\ Kristin A. Taskèn ${ }^{6,7}{ }^{(}$, Viktor Berge ${ }^{6,8}$, Vivi-Ann Solhaug ${ }^{8}$, Helmut Klocker ${ }^{9}{ }^{\circ}$, T. Brendan Murphy ${ }^{10}(\mathbb{0}$ \\ and R. William Watson ${ }^{2}$
}

Citation: Moghaddam, S.; Jalali, A.; O’Neill, A.; Murphy, L.; Gorman, L.; Reilly, A.-M.; Heffernan, Á.; Lynch, T.; Power, R.; O'Malley, K.J.; et al. Integrating Serum Biomarkers into Prediction Models for Biochemical Recurrence Following Radical Prostatectomy. Cancers 2021, 13, 4162. https://doi.org/10.3390/ cancers13164162

Academic Editor: Constantin N. Baxevanis

Received: 15 July 2021

Accepted: 14 August 2021

Published: 19 August 2021

Publisher's Note: MDPI stays neutral with regard to jurisdictional claims in published maps and institutional affiliations.

Copyright: (C) 2021 by the authors. Licensee MDPI, Basel, Switzerland. This article is an open access article distributed under the terms and conditions of the Creative Commons Attribution (CC BY) license (https:// creativecommons.org/licenses/by/ $4.0 /)$.
1 School of Mathematical Sciences, University College Cork, T12XF62 Cork, Ireland

2 UCD School of Medicine, Conway Institute of Biomolecular and Biomedical Research, UCD, D04V1W8 Dublin 4, Ireland; Amanda.oneill@ucd.ie (A.O.); lisa.murphy24@mail.dcu.ie (L.M.); laura.gorman@ucdconnect.ie (L.G.); annemarie.reilly44@mail.dcu.ie (A.-M.R.); aine.heffernan@ucdconnect.ie (Á.H.); william.watson@ucd.ie (R.W.W.)

3 Department of Urology, Trinity College, St James Hospital, D08 W9RT Dublin 8, Ireland; tlynch@stjames.ie

4 Department of Urology, Royal College of Surgeons in Ireland, Beaumont Hospital, D09V2N0 Dublin 9, Ireland; rpower@rcsi.ie

5 Department of Urology, University College Dublin, Mater Misericordiae University Hospital, D07YH5R Dublin 7, Ireland; kiaranomalley@mater.ie

6 Institute of Clinical Medicine, University of Oslo, 0318 Oslo, Norway; k.a.tasken@medisin.uio.no (K.A.T.); viktor.berge@medisin.uio.no (V.B.)

7 Department of Tumor Biology, Oslo University Hospital, 0379 Oslo, Norway

8 Department of Urology, Oslo University Hospital, 0379 Oslo, Norway;

Vivi-Ann.Solhaug@nordlandssykehuset.no

9 Department of Urology, Medical University of Innsbruck, 6020 Innsbruck, Austria; helmut.klocker@i-med.ac.at

10 UCD School of Mathematics and Statistics, University College Dublin, D04V1W8 Dublin 4, Ireland; brendan.murphy@ucd.ie

* Correspondence: shirin.moghaddam@ucc.ie (S.M.); amir.jalali@ucc.ie (A.J.)

+ These authors contributed equally to this work.

Simple Summary: Treatment decisions represent a significant dilemma for patients diagnosed with prostate cancer. The prediction of early treatment failure would inform appropriate decision making and allow the clinician and patient to consider appropriate primary treatments and adjuvant therapies. We have developed and validated a serum biomarker-based model for predicting risk of biochemical reoccurrence in prostate cancer after radical prostatectomy. This study shows that the pre-operative biomarker PEDF can improve the accuracy of the clinical factors to predict risk of biochemical reoccurrence. PEDF has anti-inflammatory effects impacting on cytokine production. This non-invasive tool can be employed prior to treatment and demonstrates significant benefit over current clinical practice, impacting on patients' outcomes and quality of life.

Abstract: This study undertook to predict biochemical recurrence (BCR) in prostate cancer patients after radical prostatectomy using serum biomarkers and clinical features. Three radical prostatectomy cohorts were used to build and validate a model of clinical variables and serum biomarkers to predict BCR. The Cox proportional hazard model with stepwise selection technique was used to develop the model. Model evaluation was quantified by the AUC, calibration, and decision curve analysis. Cross-validation techniques were used to prevent overfitting in the Irish training cohort, and the Austrian and Norwegian independent cohorts were used as validation cohorts. The integration of serum biomarkers with the clinical variables (AUC $=0.695$ ) improved significantly the predictive ability of BCR compared to the clinical variables (AUC $=0.604)$ or biomarkers alone (AUC $=0.573)$. This model was well calibrated and demonstrated a significant improvement in the predictive ability in the Austrian and Norwegian validation cohorts (AUC of 0.724 and 0.606), compared to the clinical model (AUC of 0.665 and 0.511). This study shows that the pre-operative biomarker PEDF can improve the accuracy of the clinical factors to predict BCR. This model can be employed prior to 
treatment and could improve clinical decision making, impacting on patients' outcomes and quality of life.

Keywords: biochemical recurrence; calibration; Cox model; discrimination; model evaluation; prediction models; prostate cancer; cytokine

\section{Introduction}

Radical prostatectomy (RP) represents a curative intent for localised prostate cancer; however, $20-30 \%$ of patients will fail, developing biochemical recurrence (BCR) [1]. The pre-treatment prediction of early treatment failure would inform appropriate decision making and allow the clinician and patient to consider alterative primary treatments and adjuvant therapies. Current clinical variables to inform outcome are pre-treatment PSA, biopsy Gleason Score (bxGS) and clinical stage, but additional biomarkers are needed to improve on their prediction. Commercially available tests for the prediction of BCR include Oncotype DX, Prolaris and Decipher, but these are dependent on tissue biopsies, introducing a sampling error $[2,3]$.

Serum or urine biomarkers would overcome this and represent a less invasive approach to predicting BCR. To date, limited studies have identified serum biomarkers for predicting BCR. Svatek et al. has identified preoperative biomarkers that improve the accuracy of standard models associated with BCR after RP [4]. This supports the concept that panels of blood-based proteins could improve on the prediction of BCR.

Prediction models have been widely used to aid clinical decision making. They mainly are mathematical equations or algorithms that assign a level of risk using patient variables. There are a range of time-to-event models that predict the duration of time expected before an event (e.g., recurrence of a disease) occurs rather than predicting the risk of the event at a specific time point (e.g., in logistic regression). The Cox proportional hazards model [5] is the most commonly used regression model for modelling time-to-event data.

In our current study we undertook to investigate the ability of a panel of biomarkers to predict BCR and build a model integrating the best biomarkers with the current clinical variables that significantly improved on the current clinical decision-making tools to predict 3- and 5-year BCR. These biomarkers were selected from previous discovery studies carried out in our laboratory [6] and have evidence for a role in cancer development. Insulin-Like Growth Factor Binding Protein-3 (IGFBP-3) is a p53 tumour suppressor-regulated protein and binds to IGF1, facilitating its transport in the circulation. Disruption of IGFBP-3 at the transcriptional and post-translational levels has been implicated in the pathophysiology of many cancers, including that of the prostate [7]. Apolipoprotein A-II (APOAII) has been shown to be associated with pancreatic cancer [8]. APOAII is overexpressed in prostate cancers and may be involved in cell proliferation and apoptosis [9]. CD14 is an integral part of the innate immune system. CD14-positive tumours were shown to be more vascularised in certain cancers, such as bladder cancer [10]. It has also been shown to be released into the circulation and promote tumour-related inflammation. Vitamin D-Binding Protein (VDBP/Gc-Globulin) binds Vitamin D and has been shown to increase the risk of prostate cancer [11]. Zinc $\alpha 2$-glycoprotein (ZAG) has been identified by proteomics in prostate tissue and urine, showing different concentrations in patients with prostate cancer and benign prostate hyperplasia [12]. Pigment Epithelium-Derived Factor (PEDF) has been identified as a mediator of inflammation and regulates macrophage activation [13] It has been shown to stimulate macrophages to release tumour necrosis factor and interleukin-1 [13] as well as inducing the migration of monocytes and macrophages [14], contributing to the maintenance of chronic inflammation. Decreased Tumour-Associated Macrophages is associated with prostate cancer progression [15]. Loss of PEDF could lead to reduced immunological effects and lead to the progression of the tumour, leading to BCR. 
The developed model was then internally and externally validated to evaluate its predictive performance and avoid overfitting [16].

\section{Patients and Methods}

\subsection{Patient Population and Sample Collection}

Three radical prostatectomy (RP) cohorts were used (Table 1). The Norwegian cohort was a selected group of patients consisting of an equal number of organ-confined and nonorgan-confined samples and Gleason 6,7 and 8. The majority of men in all three cohorts were Caucasian; no patients received neoadjuvant hormonal therapy or had a prior history of pelvic radiotherapy, which were the exclusion criteria. The inclusion criteria for this study included the availability of a preoperative serum sample, PSA, transrectal ultrasound guided needle biopsy pathology reports and the corresponding radical prostatectomy pathology report. BCR was defined as two consecutive PSA values $>0.4 \mathrm{ng} / \mathrm{mL}$, which correlate best with clinical progression, metastasis and need for adjuvant therapy [17].

Table 1. Clinical characteristics of the patients by cohorts, and the univariate $p$-value for each clinical variable ${ }^{\mathrm{a}}$.

\begin{tabular}{|c|c|c|c|c|}
\hline Features & Irish & Austrian & Norwegian & $p$-Value ${ }^{\mathrm{b}}$ \\
\hline Sample size $(n=577)$ & 271 & 128 & 178 & \\
\hline \multicolumn{5}{|l|}{ Pre-op } \\
\hline $\begin{array}{c}\text { PSA } \\
\text { Mean (SD) }\end{array}$ & $8.36(4.74)$ & $5.77(4.73)$ & $11.3(7.25)$ & $<0.001$ \\
\hline $\begin{array}{c}\text { DRE } \\
\text { Normal } \\
\text { Abnormal }\end{array}$ & $\begin{array}{c}198(73 \%) \\
73(27 \%)\end{array}$ & $\begin{array}{c}107(84 \%) \\
21(16 \%)\end{array}$ & $\begin{array}{c}165(93 \%) \\
13(7 \%)\end{array}$ & $<0.001$ \\
\hline $\begin{array}{c}\text { Biopsy Gleason Score } \\
6 \\
7 \\
8 \text { and above }\end{array}$ & $\begin{array}{l}132(49 \%) \\
98(36 \%) \\
42(15 \%)\end{array}$ & $\begin{array}{l}73(55 \%) \\
47(35 \%) \\
13(10 \%)\end{array}$ & $\begin{array}{l}56(31 \%) \\
76(43 \%) \\
46(26 \%)\end{array}$ & $<0.001$ \\
\hline Post-op & & & & \\
\hline $\begin{array}{l}\text { Gleason Score } \\
6 \\
7 \\
8 \text { or above }\end{array}$ & $\begin{array}{c}92(34 \%) \\
135(51 \%) \\
40(15 \%)\end{array}$ & $\begin{array}{l}37(29 \%) \\
79(62 \%) \\
12(9 \%)\end{array}$ & $\begin{array}{l}60(34 \%) \\
59(33 \%) \\
59(33 \%)\end{array}$ & $<0.001$ \\
\hline $\begin{array}{c}\text { Stage } \\
\text { Organ-confined } \\
\text { Non-organ-confined }\end{array}$ & $\begin{array}{l}155(57 \%) \\
116(43 \%)\end{array}$ & $\begin{array}{l}78(61 \%) \\
50(39 \%)\end{array}$ & $\begin{array}{l}89(50 \%) \\
89(50 \%)\end{array}$ & 0.13 \\
\hline $\begin{array}{l}\text { Time to biochemical recurrence } \\
\qquad 3 \text { years } \\
<5 \text { years }\end{array}$ & $\begin{array}{l}15.0 \% \\
18.5 \%\end{array}$ & $\begin{array}{l}29.3 \% \\
37.8 \%\end{array}$ & $\begin{array}{l}28.7 \% \\
75.5 \%\end{array}$ & $<0.001$ \\
\hline
\end{tabular}

a Statistics presented: mean (SD) or $n$ (\%). ${ }^{\mathrm{b}}$ Statistical tests performed: Kruskal-Wallis test; chi-square test of independence; log-rank test.

Blood samples were collected within three months prior to their RP and serum was isolated and stored at $-80^{\circ} \mathrm{C}$ until analysis. All samples were transported to the Conway Institute for analysis using established standard operating procedures. See ethical approval and consent details below.

\subsection{Biomarker Measurements}

A multiplex antibody-based electrochemiluminescence assay was developed (Meso Scale Discovery (MSD), Gaitherberg, MD, USA). Custom-made plates were generated. Five serum-based biomarkers were assessed on a 5-plex, 96-well 7-Spot MSD microplate plate: APOAII (Apolipoprotein AII, Abcam; ab20903, Cambridge, UK), CD14 (Cluster Differentiation 14; RnD System DY383, Abingdon, UK), Gc-globulin (Vitamin D-Binding Protein; ThermoFisher HYB 249-01B-005, Dublin, Ireland), IGFBP-3 (Insulin-Like Growth Factor Bind- 
ing Protein 3 DuoSet ELISA; RnD System DY675, Abingdon, UK) and ZAG (Zinc-alpha-2glycoprotein; Biotechne BAF4764, Dublin, Ireland). The second panel consisted of two serumbased biomarkers: PEDF (Pigment Epithelium-Derived Factor; MSD W0060165) was assessed on the Duplex 96-well 4-Spot MSD microplate plate and PSA (prostate-specific antigen; MSD W0016950, Rockville, MA, USA), following on from previous studies in our laboratory [18]. The average detection ranges for each biomarker were APOAII $=480.2-1250,000 \mathrm{pg} / \mathrm{mL}$, CD14 $=61.93-58,500 \mathrm{pg} / \mathrm{mL}$, Gc-Globulin $=87.2-1250,000 \mathrm{pg} / \mathrm{mL}$, IGFBP-3 $=250-31,000 \mathrm{pg} / \mathrm{mL}$ and ZAG $=1613-250,000 \mathrm{pg} / \mathrm{mL}$. The average detection ranges for the 2 biomarkers were as follows: $\mathrm{PEDF}=6.21-500,000 \mathrm{pg} / \mathrm{mL}$ and PSA $=230.25-100,000 \mathrm{pg} / \mathrm{mL}$. Internal pooled reference serum samples were also run. Samples were run as previously described [18] and plates were read on an MDS plate reader (MESO Quickplex S120 microplate reader using the MSD Workbench Software V2 (Rockville, MA, USA).

\subsection{Statistical Method}

Patient information used to build the model included the clinical risk factors (PSA, Digital rectal examination (DRE) and biopsy Gleason Score (bxGS)) and serum biomarkers identified previously by our group (CD14, IGFBP-3, APOAII, Gc-globulin, ZAG and PEDF) [6]. The stepwise selection technique using R software [19] was applied to identify the serum biomarkers that could predict time-to-BCR. A Cox regression model was used to predict time-to-BCR using linear and nonlinear effects of the serum biomarkers.

The model discrimination is demonstrated using the Receiver Operator Characteristic (ROC) curve and decision curve analysis [20] at 3 and 5 years. The discriminant ability of the models was also numerically determined using the area under the ROC curve (AUC). Comparison of AUC values took place via a method described by DeLong et al. [21].

The model calibration was tested using the Greenwood-Nam-D'Agostino goodnessof-fit test [22]. Calibration plots were used to investigate how close the predicted probabilities are to the actual probabilities using Frank Harrell's method [23]. This method used resampling techniques to get bias-corrected estimates of the predicted versus observed values for Cox models.

Internal validation using the Irish cohort was performed using 10-fold cross-validation to confirm that no patient was used to both develop and test the model. External validation was performed to evaluate the model performance in the independent Austrian and Norwegian cohorts. The validation technique for the Cox models proposed by Royston [24] was used, which adjust the baseline survival function to the new cohorts. This adjustment is crucial to assess the calibration of survival probabilities in the validation datasets since the event probabilities are estimated relative to an unspecified baseline function [24].

Due to the short follow-up time for the large number of validation cases, the external validation was only investigated at 3-year BCR. A second approach investigated a combined dataset used to provide more validation sample cases. We combined the Irish, Austrian and Norwegian cohorts and randomly selected 70\% (346 samples) to generate a training set for model development, and the remaining 30\% (145 samples) to test the model using the caret $\mathrm{R}$ package [25]. This approach provides sufficient data for model development and validation at 3- and 5-year BCR. The results of the combined cohort are presented in the supplementary data and described in the results section, as additional prospective studies would be required to further validate these findings.

\section{Results}

The model for predicting BCR after RP was developed using the Irish cohort (271 samples) and validated in the independent Austrian (128 samples) and Norwegian (178 samples) cohorts. Figure 1 represents the cumulative probability of time-to-BCR by cohort. 


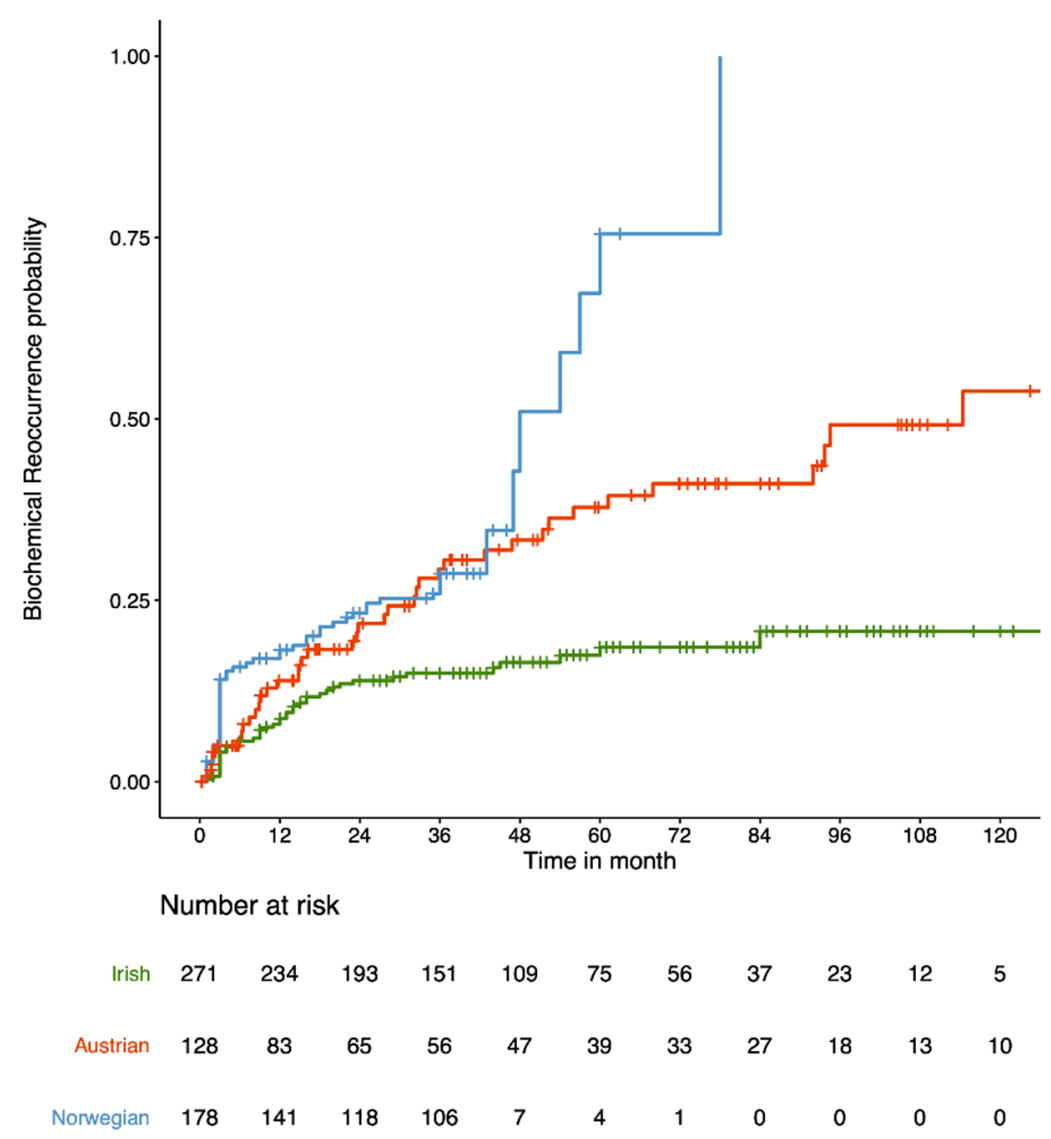

Figure 1. Cumulative probability of time-to-BCR (in months) for the 3 cohorts under investigation: Irish (green), Austrian (red) and Norwegian (blue).

The clinical characteristics grouped by cohort is shown in Table 1. The rate of 3-year BCR across the three cohorts are $15 \%$ for Irish, $29.3 \%$ for Austrian and $28.7 \%$ for Norwegian, and $18.5 \%$ for Irish, $37.8 \%$ for Austrian and $75.5 \%$ for Norwegian across the first 5 years of follow-up. It should be noted that the Norwegian cohort have a higher rate of 5-year BCR because they are a selected cohort, as previously published by Berge et al., for which the rate of 5 -year BCR is $27 \%$ [26]. The $p$-values for each clinical variable (Table 1 ) indicate significant differences in the clinical variables (except stage) between the three cohorts.

The effect of the panel of biomarkers (APOAII, CD14, Gc-globulin, IGFBP-3 and ZAG) combined with the clinical risk factors was investigated using the Cox model for the Irish cohort. The variable selection technique identified the best combination of biomarkers to integrate with either the classic three-tier ' $\mathrm{NCCN}^{\prime}$ ' risk groups (low, intermediate and high) ('NCCNbio') or the 'Clinical' variables (PSA, DRE and bxGS) ('Clinicalbio'), respectively. The effects of the risk factors on time-to-BCR for both models are given in Table 2.

The 'NCCNbio' model indicates that the intermediate-risk NCCN group and high-risk NCCN group are expected to have 1.8- and 3.1-times higher risk of developing BCR over time compared to the low-risk group, respectively. A 100,000 (pg/mL) increase in CD14 was also shown to increase the risk of BCR by $2 \%$, while a $100,000(\mathrm{pg} / \mathrm{mL})$ increase in PEDF was shown to decrease the risk of BCR by $17 \%$.

The 'Clinicalbio' model indicates that a one-unit $(\mathrm{ng} / \mathrm{mL})$ increase in the logarithm of PSA was shown to increase the risk of BCR by $163 \%$, which is approximately a $2-$ fold increase. The use of log transformation for PSA was shown to be essential in our previous studies [27], where a change in smaller values of PSA is more critical. Patients with abnormal DRE were shown to have a $23 \%$ higher risk of developing BCR over time. Moreover, patients with a bxGS of 7 and 8 were shown to have a 1.5- and 3-times higher risk of BCR compared to patients with a Gleason score of 6 , respectively. PEDF is also identified 
as an essential marker in the 'NCCNbio' model where a 100,000 (pg/mL) increase in PEDF was shown to decrease the risk of developing BCR by almost $20 \%$.

Table 2. Summary of the 'NCCNbio' and 'Clinicalbio' models developed for the Irish cohort using the hazard ratio, $95 \%$ confidence interval (CI) for the hazard ratio and $p$-value for the risk factors in the model.

\begin{tabular}{|c|c|c|c|c|c|c|}
\hline \multirow{2}{*}{ Features } & \multicolumn{3}{|c|}{ NCCNbio } & \multicolumn{3}{|c|}{ Clinicalbio } \\
\hline & Hazard Ratio & $95 \%$ CI & $p$-Value & Hazard Ratio & $95 \%$ CI & $p$-Value \\
\hline PSA $^{a}$ & - & - & - & 2.628 & $(1.45,4.75)$ & 0.001 \\
\hline $\begin{array}{c}\text { DRE } \\
\text { (Abnormal vs. normal) }\end{array}$ & - & - & - & 1.227 & $(0.62,2.43)$ & 0.556 \\
\hline $\begin{array}{c}\text { Biopsy Gleason Score } \\
(7 \text { vs. } 6) \\
(8 \text { or above vs } 6)\end{array}$ & - & - & $\begin{array}{l}- \\
-\end{array}$ & $\begin{array}{c}1.516 \\
2.99\end{array}$ & $\begin{array}{l}(0.73,3.15) \\
(1.35,6.65)\end{array}$ & $\begin{array}{l}0.265 \\
0.007\end{array}$ \\
\hline $\begin{array}{c}\text { NCCN } \\
\text { (Intermediate vs. low) } \\
\text { (High vs. low) }\end{array}$ & $\begin{array}{l}1.808 \\
3.135\end{array}$ & $\begin{array}{l}(0.72,4.54) \\
(1.33,7.39)\end{array}$ & $\begin{array}{l}0.207 \\
0.009\end{array}$ & - & - & - \\
\hline CD14 (100,000 pg/mL) & 1.02 & $(0.99,1.05)$ & 0.1 & - & - & - \\
\hline PEDF $(100,000 \mathrm{pg} / \mathrm{mL})$ & 0.831 & $(0.70,0.98)$ & 0.03 & 0.801 & $(0.68,0.95)$ & 0.009 \\
\hline
\end{tabular}

Models with the NCCN risk groups ('NCCN') and the clinical variables, namely, PSA, DRE and bxGS ('Clinical'), were used as references for the 'NCCNbio' and 'Clinicalbio' models, respectively. A model including the effect of PEDF alone ('Biomarker') was also included as an additional reference. Using cross-validation, the internal discriminate ability of both models was compared numerically (Table 3A) and graphically (Figure S1) to their references at 3- and 5-year BCR.

Table 3. The AUC for the 'NCCNbio' and 'Clinicalbio' compared to 'NCCN', 'Clinical' and 'Biomarker' for the internal validation using the Irish cohort (Panel A) and external validation using the Austrian and Norwegian cohorts (Panel B).

\begin{tabular}{|c|c|c|c|c|}
\hline \multirow[b]{2}{*}{ Models } & \multicolumn{2}{|c|}{ (A) Internal Validation } & \multicolumn{2}{|c|}{ (B) External Validation } \\
\hline & $\begin{array}{l}\text { AUC at 3-Year } \\
\text { (Irish Cohort) }\end{array}$ & $\begin{array}{l}\text { AUC at 5-Year } \\
\text { (Irish Cohort) }\end{array}$ & $\begin{array}{c}\text { AUC at 3-Year } \\
\text { (Austrian Cohort) }\end{array}$ & $\begin{array}{c}\text { AUC at 3-Year } \\
\text { (Norwegian Cohort) }\end{array}$ \\
\hline $\mathrm{NCCN}$ & 0.5335 & 0.5424 & 0.6958 & 0.5838 \\
\hline Clinical & 0.6377 & 0.6777 & 0.6971 & 0.5174 \\
\hline Biomarker & 0.5928 & 0.6236 & 0.5702 & 0.5330 \\
\hline NCCNbio & $\begin{array}{c}0.7058 ; \\
p \text {-value (vs. NCCN) < } 0.001^{\text {a }}\end{array}$ & $\begin{array}{c}0.6968 ; \\
p \text {-value (vs. } \mathrm{NCCN})=0.002^{\mathrm{a}}\end{array}$ & $\begin{array}{c}0.7065 ; \\
p \text {-value }(\text { vs. } \mathrm{NCCN})=0.901^{\mathrm{a}}\end{array}$ & $\begin{array}{c}0.6224 ; \\
p \text {-value }(\text { vs. } \mathrm{NCCN})=0.701^{\mathrm{a}}\end{array}$ \\
\hline Clinicalbio & $\begin{array}{c}0.7076 ; \\
p \text {-value }(\text { vs. Clinical })=0.024^{\text {a }}\end{array}$ & $\begin{array}{c}0.7531 ; \\
p \text {-value }\left(\text { vs. Clinical) }=0.032^{\text {a }}\right.\end{array}$ & $\begin{array}{c}0.7659 ; \\
p \text {-value }(\text { vs. Clinical })=0.034^{a}\end{array}$ & $\begin{array}{c}0.5877 ; \\
p \text {-value (vs. Clinical) }=0.042^{a}\end{array}$ \\
\hline
\end{tabular}

The AUC for the 3- and 5-year BCR (Table 3A) for the 'NCCNbio' model (AUC of 0.7058 and 0.6968 ) demonstrates a significant improvement over the 'NCCN' (AUC of 0.5335 and 0.5424 ) model alone. The 'Clinicalbio' model (AUC of 0.7076 and 0.7531) also demonstrates a significant improvement over 'Clinical' (AUC of 0.6377 and 0.6777) at 3 and 5-year BCR. The ROC (Figure S1A) and decision curves (Figure S1B) show that the integration of the biomarkers with the clinical factors not only increased sensitivity and specificity in almost all thresholds but also provided additional clinical benefit.

The Greenwood-Nam-D'Agostino test for 'NCCNbio' ( $p$-value of 0.369 at 3 years and 0.610 at 5 years) and 'Clinicalbio' ( $p$-value of 0.897 at 3 years and 0.691 at 5 years) indicate that both models are well calibrated (Figure S1C). 
Using the independent Austrian and Norwegian cohorts, the external discrimination ability of the models compared to their references at 3 years is shown in Table 3B and Figure 2. The AUC of the 'NCCNbio' model (0.7065 and 0.6224) has no significant improvement over 'NCCN' (AUC of 0.6958 and 0.5838) for the Austrian and Norwegian cohorts, respectively (Table $3 \mathrm{~B}$ ). However, the integration of the biomarker with clinical factors in the 'Clinicalbio' model (AUC of 0.7659 and 0.5877) demonstrated a significant improvement over 'Clinical' (AUC of 0.6971 and 0.5174). The ROC and decision curves for the Austrian cohort on the top of Figure 2A,B demonstrates that the integration of PEDF with the clinical risk factors ('Clinicalbio') increased sensitivity and specificity in almost all thresholds and also provided additional clinical benefits (compared to the 'Clinical' model).
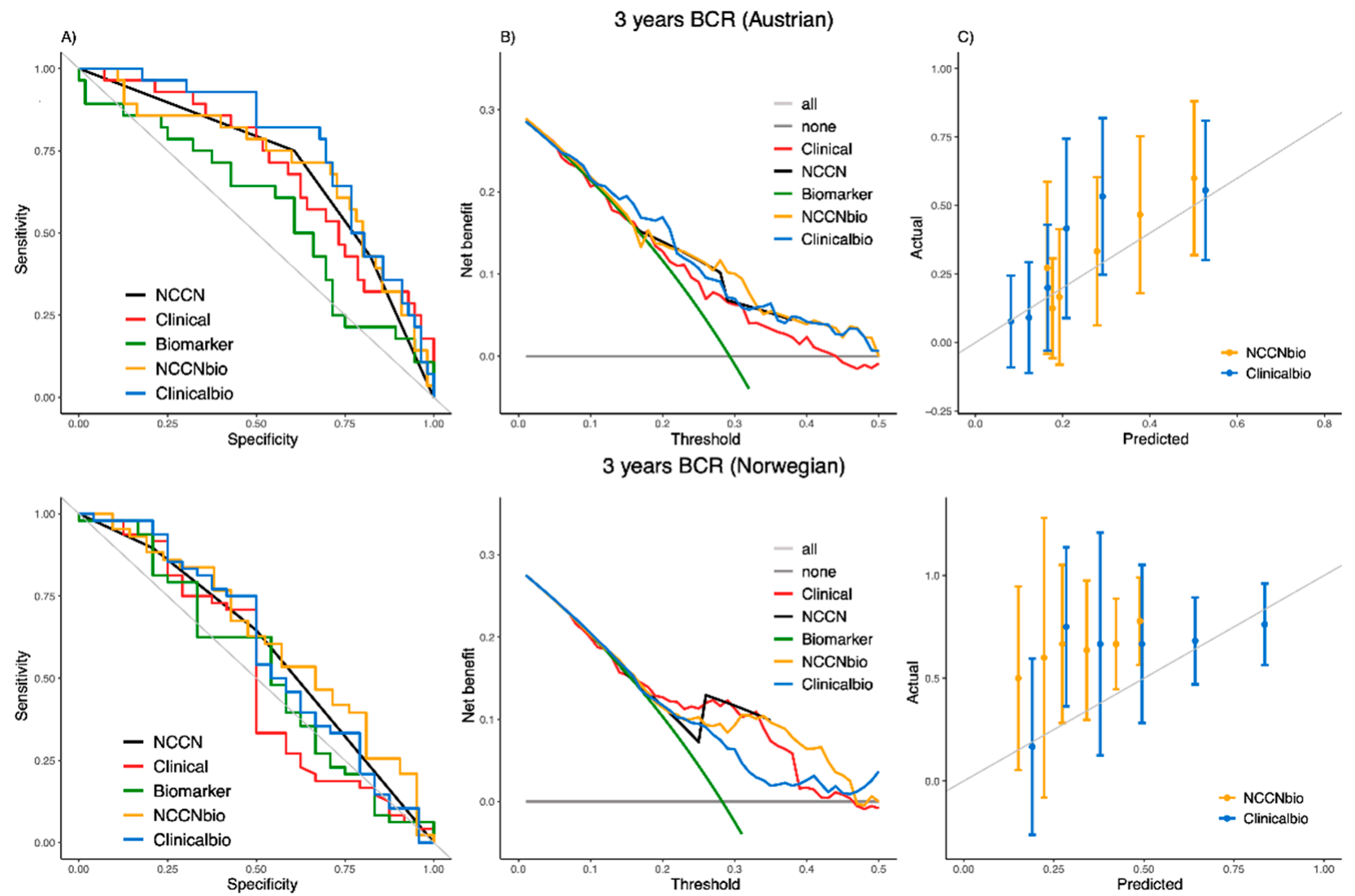

Figure 2. ROC curve (A), decision curve (B) and calibration plot (C) of the 'NCCNbio' and 'Clinicalbio' models externally compared with 'NCCN', 'Clinical' and 'Biomarker' models in independent Austrian (on the top) and Norwegian (on the bottom) cohorts at 3 years.

The Greenwood-Nam-D'Agostino test for 'NCCNbio' ( $p$-value of 0.286 of Austrian and 0.008 of Norwegian) and 'Clinicalbio' ( $p$-value of 0.121 of Austrian and $<0.001$ of Norwegian) shows that both models are well calibrated to predict BCR for the Austrian cohort but not for the Norwegian cohort. The calibration plots (Figure 2C) show that both models generally give an overestimate for the chance of time-to-BCR at 3 years for the Norwegian cohort.

We also undertook to combine the three cohorts (Irish, Austrian and Norwegian) to give a larger sample size and build a separate model for predicting BCR. The model was developed using $70 \%$ of the combined cohort (405 samples) and validated using the remaining $30 \%$ of the combined cohort (172 samples). The clinical characteristics are shown in Table S1. The $p$-values of the combined cohort in Table S1 show no significant differences in clinical variables between test and train set (except for 'Gleason Grade'). 
The model summaries for the combination of the selected biomarkers with either the classic three-tier 'NCCN' risk groups ('NCCNbio-s') or the 'Clinical' variables ('Clinicalbio$\mathrm{s}^{\prime}$ ) are given in Table S2.

The discrimination ability of the combined cohort is shown in Table S3A. This demonstrates a significant improvement in the AUC values for 'NCCNbio-s' (AUC of 0.7329 and 0.7601 ) in comparison to 'NCCN-s' and 'Clinicalbio-s' (AUC of 0.7713 and 0.8332) in comparison to 'Clinical-s' for both 3- and 5-year BCR. The ROC and decision curves for the combined cohort in Figure S2 show that the integration of the biomarkers with clinical factors has increasing sensitivity and specificity and provides additional clinical benefit. The calibration plots also indicate that 'NCCNbio-s' ( $p$-value of 0.168 and 0.457 ) and 'Clinicalbio-s' ( $p$-value of 0.856 and 0.716 ) are well calibrated at 3 and 5 years.

The external validation of the combined cohort also demonstrates improved discrimination ability. The ROC and decision curves for the test set in Figure S3 show that the integration of PEDF (which was the biomarker identified using the Irish cohort to build the model and then validated in the Austrian and Norwegian cohorts, as above) into the clinical risk factors ('Clinicalbio-s') increased the sensitivity and specificity, and also provided additional clinical benefit. The Greenwood-Nam-D'Agostino test results for 'NCCNbio-s' ( $p$-value of 0.335 and 0.107 ) and 'Clinicalbio-s' ( $p$-value of 0.258 and 0.055 ) indicate that both models are calibrated in the test set at 3 and 5 years (Figure S3).

\section{Discussion}

We have developed statistical models that combine biomarkers into the clinical variables to predict BCR. The clinical information was considered on their own (PSA, DRE and bxGS) and also combined into the 3-tier NCCN [28], so they can be used as a tool for treatment decision making.

The use of survival methods and the Cox model [5] were appropriate modelling approaches. However, the initial analysis using logistic regression for 3-year and 5-year BCR [29] identified PEDF as the important marker, which was in line with the finding of the Cox model.

The methodology for validation of the Cox models was not straightforward as would be for logistic models. This is mainly because the un-estimated baseline survival in the Cox model is a vital component for the external validation. Royston [24] proposed an approach to tackle this issue, which was used for our external validation.

Traditional biomarker studies build a model in one cohort and validate in an independent cohort [30], which was the approach we undertook. However, due to the lack of 5-year follow-up in the individual cohort approach, we also undertook a combination of the cohorts to generate a combined-cohort approach. Pooling data across the cohorts helps to achieve a larger sample cohort, which allowed model validation at 5-year BCR [31,32]; however, additional prospective studies of these biomarkers will be required.

Our study demonstrated that the combination of PEDF with PSA, DRE and bxGS improved the prediction of post-operative BCR in both the individual cohort and combinedcohort studies. Previous studies by our group and others have demonstrated that the integration of serum biomarkers improves prediction models [33-35]. PEDF has multiple biological actions and is expressed by prostate epithelial and stromal cells [36]. Downregulation of PEDF expression in prostate cancer has been linked to poor prognosis [37] and the tumours metastatic phenotype [38]. It has been identified as a major antimetastatic factor [39], which supports its role as a predictor of disease progression.

Robust methods, including the ROC curve, calibration and decision curve analysis, were used to access model performance. The discrimination ability of the models is presented at different thresholds; however, an optimal threshold needs to be chosen to make the best clinical decision in practice. The selection of this threshold could be challenging as it depends on a trade-off between a more sensitive or a more specific test [40]. Further validation studies will be required to identify the best clinically accepted thresholds. 


\section{Conclusions}

This study shows that the pre-operative biomarker PEDF can improve the accuracy of the clinical factors to predict BCR. The clinical and serum biomarker model can be employed prior to treatment and could improve clinical decision making for the physician and patient to choose the appropriate treatment, and this could impact on patients' outcomes and quality of life.

Supplementary Materials: The following are available online at https:/ /www.mdpi.com/article/10 .3390 / cancers13164162/s1, Figure S1: ROC curve (A), decision curve (B) and calibration plot (C) of the 'NCCNbio' and 'Clinicalbio' models internally compared with 'NCCN', 'Clinical' and 'Biomarker' models in the Irish cohort at 3-year (on the top) and 5-year (on the bottom), Figure S2: ROC curve (A), decision curve (B) and calibration plot (C) of the 'NCCNbio-s' and 'Clinicalbio-s' models internally compared with 'NCCN', 'Clinical' and 'Biomarker' models in the train set at 3-year (on the top) and 5-year (on the bottom), Figure S3: ROC curve (A), decision curve (B) and calibration plot (C) of the 'NCCNbio-s' and 'Clinicalbio-s' models externally compared with ' $\mathrm{NCCN}^{\prime}$, 'Clinical' and 'Biomarker' models in independent test set at 3-year (on the top) and 5-year (on the bottom), Table S1: Clinical characteristics of the patients in the train and test sets including the univariate $p$-value for each clinical variablea, Table S2: Summary of the 'NCCNbio-s' and 'Clinicalbio-s' models developed on the train set using hazard ratio, $95 \%$ confidence interval (CI) for hazard ratio and $p$-value for each risk factor in the model, Table S3: The AUC for the 'NCCNbio-s' and 'Clinicalbio-s' compared to 'NCCN-s', 'Clinical-s' and 'Biomarker-s' for internal validation using the Train set (panel A) and external validation using the Test set (panel B).

Author Contributions: Conceptualization, S.M., A.J., T.B.M. and R.W.W.; data curation, A.O., L.M., L.G., A.-M.R., Á.H., T.L., R.P., K.J.O., K.A.T., V.B., V.-A.S. and H.K.; formal analysis, S.M., A.J., T.B.M. and R.W.W.; funding acquisition, K.A.T. and R.W.W.; methodology, S.M., A.J., T.B.M. and R.W.W.; project administration, A.O., L.M., L.G., A.-M.R. and Á.H.; software, S.M. and A.J.; validation, S.M., A.J. and R.W.W.; visualization, S.M. and A.J.; writing — original draft, S.M., A.J. and R.W.W.; writingreview and editing, S.M., A.J., T.B.M. and R.W.W. All authors have read and agreed to the published version of the manuscript.

Funding: This publication has emanated from research conducted with the financial support of Science Foundation Ireland (SFI) under Grant Number 15/IA/3104. The Prostate Biobank (REC2013/1713) from Oslo University Hospital. Irish Cancer Society (PCI11WAT) for the Prostate Cancer Research Consortium biocollection.

Institutional Review Board Statement: Sample collection and processing were ethically approved by the IRBs at Beaumont Hospital, St James Hospital and Mater Misericordiae University Hospital in Ireland, Innsbruck Medical University (2018/1072) in Austria and Prostate Cancer Biobank (2013/1713) at Oslo University Hospital, Norway. The Human Research Ethical committee at University College Dublin also approved the study (LS-E-11-39). The patient information leaflet and consent form were written and constructed in line with best practice and the EU Data Protection Directive and Data protection Acts 1988 and 2018, and all patients gave written informed consent to participate in the study. All steps were carried out in accordance with national guidelines and regulations as well as the Declaration of Helsinki.

Informed Consent Statement: Informed consent was obtained from all subjects involved in the study.

Data Availability Statement: The data are available to other researchers on written request to the corresponding author.

Acknowledgments: We acknowledge all the patients involved in this study.

Conflicts of Interest: The authors declare no conflict of interest. The funders had no role in the design of the study; in the collection, analyses, or interpretation of data; in the writing of the manuscript, or in the decision to publish the results. 


\section{References}

1. Tourinho-Barbosa, R.; Srougi, V.; Nunes-Silva, I.; Baghdadi, M.; Rembeyo, G.; Eiffel, S.S.; Barret, E.; Rozet, F.; Galiano, M.; Cathelineau, X.; et al. Biochemical recurrence after radical prostatectomy: What does it mean? Int. Braz. J. Urol. 2018, 44, 14-21. [CrossRef]

2. Carneiro, A.; Priante Kayano, P.; Gomes Barbosa, Á.R.; Langer Wroclawski, M.; Ko Chen, C.; Cavlini, G.C.; Reche, G.J.; SanchezSalas, R.; Tobias-Machado, M.; Sowalsky, A.G.; et al. Are localized prostate cancer biomarkers useful in the clinical practice? Tumor Biol. 2018, 40. [CrossRef]

3. Murphy, L.; Prencipe, M.; Gallagher, W.M.; Watson, R.W. Commercialized biomarkers: New horizons in prostate cancer diagnostics. Expert Rev. Mol. Diagn. 2015, 15, 491-503. [CrossRef]

4. Svatek, R.S.; Jeldres, C.; Karakiewicz, P.I.; Suardi, N.; Walz, J.; Roehrborn, C.G.; Montorsi, F.; Slawin, K.M.; Shariat, S.F. Pretreatment biomarker levels improve the accuracy of post-prostatectomy nomogram for prediction of biochemical recurrence. Prostate 2009, 69, 886-894. [CrossRef]

5. Cox, D.R. Regression models and life-tables. J. R. Stat. Soc. Ser. B Methodol. 1972, 34, 187-202. [CrossRef]

6. Boyce, S.; Fan, Y.; Watson, R.W.; Murphy, T.B. Evaluation of prediction models for the staging of prostate cancer. BMC Med Inform. Decis. Mak. 2013, 13, 126. [CrossRef]

7. Cai, Q.; Dozmorov, M.; Oh, Y. IGFBP-3/IGFBP-3 receptor system as an anti-tumor and anti-metastatic signaling in cancer. Cells 2020, 9, 1261. [CrossRef]

8. Honda, K.; Kobayashi, M.; Okusaka, T.; Rinaudo, J.A.; Huang, Y.; Marsh, T.; Sanada, M.; Sasajima, Y.; Nakamori, S.; Shimahara, M.; et al. Plasma biomarker for detection of early stage pancreatic cancer and risk factors for pancreatic malignancy using antibodies for apolipoprotein-AII isoforms. Sci. Rep. 2015, 5, 1-5. [CrossRef]

9. Malik, G.; Ward, M.D.; Gupta, S.K.; Trosset, M.W.; Grizzle, W.E.; Adam, B.L.; Diaz, J.I.; Semmes, O.J. Serum levels of an isoform of apolipoprotein A-II as a potential marker for prostate cancer. Clin. Cancer Res. 2005, 11, 1073-1085.

10. Cheah, M.T.; Chen, J.Y.; Sahoo, D.; Contreras-Trujillo, H.; Volkmer, A.K.; Scheeren, F.A.; Volkmer, J.P.; Weissman, I.L. CD14expressing cancer cells establish the inflammatory and proliferative tumor microenvironment in bladder cancer. Proc. Natl. Acad. Sci. USA 2015, 112, 4725-4730. [CrossRef]

11. Zhang, Q.; Shan, Y. Genetic polymorphisms of vitamin D receptor and the risk of prostate cancer: A meta-analysis. J. BUON 2013, 18, 961-969.

12. Katafigiotis, I.; Tyritzis, S.I.; Stravodimos, K.G.; Alamanis, C.; Pavlakis, K.; Vlahou, A.; Makridakis, M.; Katafigioti, A.; Garbis, S.D.; Constantinides, C.A. Zinc $\alpha 2$-glycoprotein as a potential novel urine biomarker for the early diagnosis of prostate cancer. BJU Int. 2012, 110, E688-E693. [CrossRef] [PubMed]

13. Chavan, S.S.; Hudson, L.K.; Li, J.H.; Ochani, M.; Harris, Y.; Patel, N.B.; Katz, D.; Scheinerman, J.A.; Pavlov, V.A.; Tracey, K.J. Identification of Pigment Epithelium-Derived Factor as an adipocyte-derived inflammatory factor. Mol. Med. 2020, 18, 1161-1168. [CrossRef] [PubMed]

14. Nelius, T.; Samathanam, C.; Martinez-Marin, D.; Gaines, N.; Stevens, J.; Hickson, J.; de Riese, W.; Filleur, S. Positive correlation between PEDF expression levels and macrophage density in the human prostate. Prostate 2013, 73, 549-561. [CrossRef]

15. Shimura, S.; Yang, G.; Ebara, S.; Wheeler, T.M.; Frolov, A.; Thompson, T.C. Reduced infiltration of tumor-associated macrophages in human prostate cancer: Association with cancer progression. Cancer Res. 2000, 60, 5857-5861.

16. Steyerberg, E.W.; Harrell, F.E. Prediction models need appropriate internal, internal-external, and external validation. J. Clin. Epidemiol. 2016, 69, 245-247. [CrossRef] [PubMed]

17. Stephenson, A.J.; Kattan, M.W.; Eastham, J.A.; Dotan, Z.A.; Bianco, F.J., Jr.; Lilja, H.; Scardino, P.T. Defining biochemical recurrence of prostate cancer after radical prostatectomy: A proposal for a standardized definition. J. Clin. Oncol. 2006, 24, $3973-3978$. [CrossRef]

18. Oon, S.F.; Fanning, D.M.; Fan, Y.; Boyce, S.; Murphy, T.B.; Fitzpatrick, J.M.; Watson, R.W. The identification and internal validation of a preoperative serum biomarker panel to determine extracapsular extension in patients with prostate cancer. Prostate 2012, 72, 1523-1531. [CrossRef]

19. R Core Team. R: A Language and Environment for Statistical Computing; R Foundation for Statistical Computing: Vienna, Austria, 2013; Available online: http:/ / www.R-project.org/ (accessed on 3 April 2013).

20. Vickers, A.J.; Elkin, E.B. Decision curve analysis: A novel method for evaluating prediction models. Med. Decis. Making. 2006, 26, 565-574. [CrossRef]

21. DeLong, E.R.; DeLong, D.M.; Clarke-Pearson, D.L. Comparing the areas under two or more correlated receiver operating characteristic curves: A nonparametric approach. Biometrics 1988, 837-845. [CrossRef]

22. D'Agostino, R.B.; Nam, B.H. Evaluation of the performance of survival analysis models: Discrimination and calibration measures. Handb. Stat. 2003, 23, 1-25.

23. Harrell, F.E., Jr. rms: Regression Modeling Strategies. R Package Version 5.1-2. 2018. Available online: https://CRAN.R-project. org / package $=$ rms (accessed on 7 January 2018).

24. Royston, P.; Altman, D.G. External validation of a Cox prognostic model: Principles and methods. BMC Med. Res. Methodol. 2013, 13, 33. [CrossRef]

25. Kuhn, M. Building predictive models in R using the caret package. J. Stat. Softw. 2008, 28, 1-26. [CrossRef] 
26. Berge, V.; Berg, R.E.; Hoff, J.R.; Wessel, N.; Svindland, A.; Karlsen, S.J.; Eri, L.M. Five-year progression-free survival in 577 patients operated on with laparoscopic radical prostatectomy for localized prostate cancer. Scand. J. Urol. Nephrol. 2012, 46, 8-13. [CrossRef]

27. Jalali, A.; Foley, R.W.; Maweni, R.M.; Murphy, K.; Lundon, D.J.; Lynch, T.; Power, R.; O’Brien, F.; O’Malley, K.J.; Galvin, D.J.; et al. A risk calculator to inform the need for a prostate biopsy: A rapid access clinic cohort. BMC Med. Inform. Decis. Making. 2020, 20, 148. [CrossRef] [PubMed]

28. Mohler, J.L.; Armstrong, A.J.; Bahnson, R.R.; D'Amico, A.V.; Davis, B.J.; Eastham, J.A.; Enke, C.A.; Farrington, T.A.; Higano, C.S.; Horwitz, E.M.; et al. Prostate cancer, version 1.2016. J. Natl. Compr. Cancer Network. 2016, 14, 19-30. [CrossRef] [PubMed]

29. Moghaddam, S.; Murphy, L.; Reilly, A.M.; Heffernan, Á.; Lynch, T.; Power, R.; O’Malley, J.K.; Taskèn, K.A.; Berge, V.; Solhaug, V.; et al. Logistic Regression Models to Predict Biochemical Reoccurrence in Prostate Cancer Patients. In Proceedings of the 39th Conference on Applied Statistics, Dublin, Ireland, 15-17 May 2019.

30. Lin, D.W.; Crawford, E.D.; Keane, T.; Evans, B.; Reid, J.; Rajamani, S.; Brown, K.; Gutin, A.; Tward, J.; Scardino, P.; et al. Identification of men with low-risk biopsy-confirmed prostate cancer as candidates for active surveillance. Urol. Oncol. 2018, 36, 310.e7-310.e13. [CrossRef] [PubMed]

31. Connell, S.P.; Yazbek-Hanna, M.; McCarthy, F.; Hurst, R.; Webb, M.; Curley, H.; Walker, H.; Mills, R.; Ball, R.Y.; Sanda, M.G.; et al. A four-group urine risk classifier for predicting outcomes in patients with prostate cancer. BJU Int. 2019, 124, 609-620. [CrossRef] [PubMed]

32. Klocker, H.; Golding, B.; Weber, S.; Steiner, E.; Tennstedt, P.; Keller, T.; Schiess, R.; Gillessen, S.; Horninger, W.; Steuber, T. Development and validation of a novel multivariate risk score to guide biopsy decision for the diagnosis of clinically significant prostate cancer. BJUI Compass. 2020, 1, 15-20. [CrossRef]

33. Murphy, K.; Murphy, B.T.; Boyce, S.; Flynn, L.; Gilgunn, S.; O’Rourke, C.J.; Rooney, C.; Stöckmann, H.; Walsh, A.L.; Finn, S.; et al. Integrating biomarkers across omic platforms: An approach to improve stratification of patients with indolent and aggressive prostate cancer. Mol. Oncol. 2018, 12, 1513-1525. [CrossRef]

34. Foley, R.W.; Gorman, L.; Sharifi, N.; Murphy, K.; Moore, H.; Tuzova, A.V.; Perry, A.S.; Murphy, T.B.; Lundon, D.J.; Watson, R.W.G. Improving multivariable prostate cancer risk assessment using the Prostate Health Index. BJU Int. 2016, 117, 409-417. [CrossRef]

35. Jalali, A.; Kitching, M.; Martin, K.; Richardson, C.; Murphy, T.B.; FitzGerald, S.P.; Watson, R.W.; Perry, A.S. Integrating inflammatory serum biomarkers into a risk calculator for prostate cancer detection. Sci. Reports. 2021, 11, 1-8.

36. Filleur, S.; Nelius, T.I.; De Riese, W.; Kennedy, R.C. Characterization of PEDF: A multi-functional serpin family protein. J. Cell. Biochem. 2009, 106, 769-775. [CrossRef]

37. Doll, J.A.; Stellmach, V.M.; Bouck, N.P.; Bergh, A.R.; Lee, C.; Abramson, L.P.; Cornwell, M.L.; Pins, M.R.; Borensztajn, J.; Crawford, S.E. Pigment epithelium-derived factor regulates the vasculature and mass of the prostate and pancreas. Nat. Med. 2003, 9, 774-780. [CrossRef]

38. Halin, S.; Wikström, P.; Rudolfsson, S.H.; Stattin, P.; Doll, J.A.; Crawford, S.E.; Bergh, A. Decreased pigment epithelium-derived factor is associated with metastatic phenotype in human and rat prostate tumors. Cancer Res. 2004, 64, 5664-5671. [CrossRef] [PubMed]

39. Becerra, S.P.; Notario, V. The effects of PEDF on cancer biology: Mechanisms of action and therapeutic potential. Nat. Rev. Cancer. 2013, 13, 258-271. [CrossRef]

40. Vickers, A.J.; van Calster, B.; Steyerberg, E.W. A simple, step-by-step guide to interpreting decision curve analysis. Diagn. Progn. Res. 2019, 3, 1-8. [CrossRef] [PubMed] 